\title{
Tornado's Compressibility
}

\author{
Kern E. Kenyon \\ 4632 North Lane, Del Mar, USA
}

Correspondence to: Kern E. Kenyon, kernken@aol.com

Keywords: Tornado, Compressibility

Received: March 23, $2020 \quad$ Accepted: April 11, $2020 \quad$ Published: April 14, 2020

Copyright $\odot 2020$ by author(s) and Scientific Research Publishing Inc.

This work is licensed under the Creative Commons Attribution International License (CC BY 4.0).

http://creativecommons.org/licenses/by/4.0/

\section{(c) (i) Open Access}

\section{ABSTRACT}

An earlier fluid mechanical model of a tornado [1], that assumed constant air density, forecast a horizontal rotary wind speed that decreased inversely as the distance increased from the tornado's central axis. Such a velocity function implies that the force of friction is zero. However, if compressibility of the air is included in the model, and everything else is kept the same, dynamics changes the velocity structure to being constant and independent of the radius. Friction is also zero in this case. Observations are needed to find out what the wind structure is doing.

\section{INTRODUCTION}

Atmospheric pressure inside a tornado at ground level has been measured to be about $100 \mathrm{mb}$ lower than the ambient pressure outside the tornado [2]. This pressure difference occurred over a horizontal distance that is small compared to the same vertical pressure difference from the ground to the altitude of about $1 \mathrm{~km}$ under calm conditions. What is the magnitude of the compressibility involved in a tornado, and to what extent is the structure and dynamics of the rotary winds affected? Answers to these questions are given below from a model.

In the recent application of the principle of least friction to a tornado [1] a constant atmospheric density was assumed in the theory. Compressibility is normally not used in most discussions of meteorological motions, except when sound waves are treated. After all, compared to the speed of sound in air, the maximum wind speeds of a tornado $(200 \mathrm{mph})$ is a small ratio, at which investigations normally go no further.

In the present case motivation was sparked by the earlier study [1], in which the tornado model produced a rotational wind speed function that had no friction according to the Navier-Stokes equations (i.e. varying inversely as the distance from the tornado's central axis). From that result followed the notion: some persistent flow phenomena seen in nature owe their existence to the smallness of the force of friction. Wonder then set in whether or not the inclusion of compressibility of the air in the model would destroy this line of reasoning. As it turns out the wind speed structure is predicted to change, but it does so in such a way that friction is again zero. That finding was a surprise, and seems worth reporting on. 


\section{METHOD}

Three equations in three unknowns follow by adding one linear algebraic equation to an existing model [1], which contains two non-linear differential equations in two unknowns, pressure and velocity. The linear equation adds the third unknown, air density.

$$
p=C-\frac{1}{2} \rho v^{2}
$$

where $C$ is a constant, which usually depends on the streamline but here it is taken to be the same for all streamlines and in fact assumed to be zero. That assumption should cause no disruption to the dynamics. Equation (1) is Bernoulli's law applied to all closed streamlines of the horizontally rotating flow. $p$ and $v$ are atmospheric pressure and wind speed respectively.

For steady motion the cross-stream balance of forces is

$$
\frac{\mathrm{d} p}{\mathrm{~d} r}=\frac{\rho v^{2}}{r}
$$

where $\rho$ is the air density and $r$ measures distance from the model tornado's central axis (the core). On the RHS is the outward centrifugal force and on the LHS is the inward pressure force. So far Equation (1) and Equation (2) constitute two equations in three unknowns. One more equation is needed: the equation of state.

From the perfect gas (Boyel's) law comes

$$
p=K \rho
$$

where $K$ is a constant, which is the product of the gas constant $R$ for air and the temperature $T$, taken constant [3].

Between (1) and (2) the quantity $\rho v^{2}$ can be eliminated to give a differential equation for the pressure alone

$$
r \frac{\mathrm{d} p}{\mathrm{~d} r}+2 p=0
$$

The solution being

$$
p=\frac{\text { const }}{r^{2}}
$$

Then the density can be obtained from (3). Finally the velocity from (1) or (2) is found to be a constant, independent of $r$. Friction is therefore zero!

\section{CONCLUSION}

Based on a dynamic model of a tornado, it takes relatively few steps to arrive at the prediction that the compressibility of air can significantly alter the radial structure of the rotary winds from varying inversely with distance from the tornado's axis when it is absent to being a constant when present. In either case the force of friction is zero. Included in the model is Bernoulli's law along closed circular streamlines, the cross-stream force balance between the outward centrifugal force and an inward pressure gradient and Boyle's law relating air pressure and density. Available observations of tornado winds may not be able to sort out this difference in radial dependence.

\section{CONFLICTS OF INTEREST}

The author declares no conflicts of interest regarding the publication of this paper.

\section{REFERENCES}

1. Kenyon, K.E. (2020) Tornado's Shear. European International Journal of Science and Technology, In Press. 
2. Schlatter, T. (2003) Weather Queries. Weatherwise Magazine, 56, 54-56. https://doi.org/10.1080/00431670309605339

3. Brunt, D. (1952) Physical and Dynamical Meteorology. Cambridge University Press, New York, 30. 